\title{
Usando séries temporais obtidas experimentalmente como condições de contorno para a simulação numérica de trocadores de calor solo-ar
}

Using experimentally obtained time series as boundary conditions for the numerical simulation of earth-air heat exchangers

\author{
M. K. Rodrigues ${ }^{1 *}$; H. S. Gonçalves ${ }^{2}$; R. S. Brum ${ }^{3}$, J. Vaz ${ }^{2}$; L. A. O. Rocha ${ }^{4}$; \\ J. V. A. Ramalho ${ }^{3}$; E. D. dos Santos ${ }^{1,2}$; L. A. Isoldi ${ }^{1,2}$

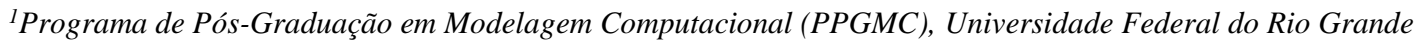 \\ (FURG) , 96.203-900, Rio Grande-RS, Brasil \\ ${ }^{2}$ Escola de Engenharia (EE), Universidade Federal do Rio Grande (FURG), 96.203-900, Rio Grande-RS, Brasil \\ ${ }^{3}$ Departamento de Matemática e Estatística (DME), Universidade Federal de Pelotas (UFPel), 96.010-900, \\ Capão do Leão-RS, Brasil \\ ${ }^{4}$ Departamento de Engenharia Mecânica (DEMEC), Universidade Federal do Rio Grande do Sul (UFRGS), \\ 90.050-170, Porto Alegre-RS, Brasil
}

*michel.professor@yahoo.com.br

(Recebido em 26 de abril de 2017; aceito em 22 de maio de 2017)

O Trocador de Calor Solo-Ar (TCSA) é um sistema que propicia redução no consumo de energia elétrica em equipamentos de condicionamento de ar tradicionais através de uma melhoria da condição térmica no interior de edificações. Dessa forma, o presente trabalho tem a finalidade de utilizar a ferramenta Boundary Profiles, disponível no software FLUENT, para promover a inserção de dados experimentais como condições de contorno para a modelagem computacional de TCSA, diferentemente do que vinha sendo feito em trabalhos anteriores encontrados na literatura. Essa proposta é uma contribuição científica importante na parte metodológica da modelagem computacional de TCSA, pois permite que dados experimentais de temperatura, ao longo do tempo, sejam introduzidos diretamente no modelo computacional, ou seja, sem a necessidade de qualquer técnica de ajuste estatístico. Com isso, os resultados mostram que os dados obtidos foram gerados de forma mais satisfatória pelo modelo computacional, observando-se reduções nos erros numéricos de até 19\%, evidenciando maiores aproximações com resultados experimentais presentes na literatura. Essa qualidade na aproximação dos resultados foi atestada através do estudo de erros numéricos, que também é uma contribuição deste trabalho.

Palavras-chave: Trocador de Calor Solo-Ar, Boundary Profiles, Modelagem Computacional.

The Earth-Air Heat Exchanger (EAHE) is a system that provides a reduction in the consumption of electric energy in traditional air conditioning equipment through an improvement of the thermal condition inside buildings. Thus, the present work has the purpose of using the Boundary Profiles tool, available in the FLUENT software, to promote the insertion of experimental data as boundary conditions for the computational modeling of EAHE, unlike what had been done in previous works found in literature. This proposal is an important scientific contribution in the methodological part of the computational modeling of EAHE, since it allows experimental temperature data, over time, to be introduced directly into the computational model, that is, without the need for any statistical adjustment technique. Therefore, the results show that the data obtained were generated in a more satisfactory way by the computational model, with reductions in numerical errors of up to $19 \%$, evidencing greater approximations with experimental results in the literature. This quality in the approximation of results was attested through the study of numerical errors, which is also a contribution of this work.

Keywords: Earth-Air Heat Exchanger, Boundary Profiles, Computational Modeling. 


\section{INTRODUÇÃO}

O Trocador de Calor Solo-Ar (TCSA) é um dispositivo constituído por um ou mais dutos enterrados no solo, por onde o ar escoa movido pela ação de ventiladores. Esse dispositivo é capaz de aproveitar a energia térmica presente na camada superficial do solo (subsolo), que é uma classe de energia renovável. Portanto, a sua principal finalidade é propiciar uma melhoria na condição térmica de edificações, visando uma diminuição no consumo de energia elétrica de equipamentos convencionais de condicionamento de ar $[1,2]$.

A literatura sobre o comportamento térmico do solo no decorrer do tempo é um importante embasamento teórico no estudo de TCSA e pode ser encontrada, por exemplo, em [3, 4, 5, 6, 7, $8,9]$. Por outro lado, importantes recomendações de leitura sobre o princípio de funcionamento do TCSA podem ser visualizadas em [10,11, 12]. Essa bibliografia inicial apresenta aspectos fundamentais para a compreensão dos fenômenos físicos presentes no estudo de TCSA, bem como princípios essenciais para sua modelagem.

Além desses trabalhos, a literatura apresenta de forma ampla uma diversidade de assuntos tratando da modelagem computacional de TCSA, como pode ser observado em $[1,2,13,14,15$, $16,17,18,19,20,21,22]$. Nesses trabalhos a modelagem computacional permitiu simular o comportamento térmico do solo, bem como o comportamento térmico e dinâmico do ar ao escoar pelo interior dos dutos. Com isso, foi possível verificar e validar os modelos computacionais estabelecidos, bem como promover distintas análises e aplicações.

Entretanto, diferentemente do que foi feito em $[1,2,13,14,15,16,17,18,20,23,24,25$, 26], o presente estudo apresenta uma nova contribuição na modelagem computacional de TCSA, na parte metodológica, com a inserção de séries temporais de temperatura, a partir de dados monitorados experimentalmente, como condições de contorno impostas ao modelo computacional. Para isso, foi realizada a verificação e validação do modelo computacional desenvolvido, considerando a implementação do recurso proposto pela ferramenta Boundary Profiles do software FLUENT.

Portanto, o principal objetivo deste trabalho é simular numericamente o comportamento térmico e fluidodinâmico de um determinado TCSA utilizando a ferramenta Boundary Profiles para impor as condições de contorno de variação de temperatura a partir de valores obtidos experimentalmente $[13,14]$.

\section{MATERIAL E MÉTODOS}

A metodologia utilizada neste estudo é conhecida na literatura como dinâmica dos fluidos computacional (CFD - Computational Fluid Dynamics). Essa ferramenta se baseia na discretização do domínio computacional em um número finito de células e na aplicação de um método numérico adequado para a solução do problema [27, 28].

Para tanto, foram empregados os softwares GAMBIT (v. 2.4.6), FLUENT (v. 6.3.26) e EXCEL (v. 2010). Também é integrante dessa metodologia o modelo computacional simplificado de TCSA [15], validado e verificado com base nos resultados obtidos em [13, 14]. A presente metodologia apresentada vem sendo utilizada pelo grupo de pesquisa do programa de pós-graduação em modelagem computacional da FURG e seus colaboradores nos estudos de TCSA, gerando resultados satisfatórios e presentes na literatura de referência.

\subsection{Descrição do problema}

A configuração geométrica do TCSA estudado neste trabalho é caracterizada por três dutos retilíneos (dispostos em forma de um arranjo triangular) inseridos no subsolo (Figura 1). O modelo apresentado é uma simplificação do modelo completo descrito em [13]. Além disso, o domínio computacional tem as seguintes dimensões para o volume de solo estudado: $H_{s}$ (profundidade), $W_{s}$ (largura) e $L_{s}$ (comprimento), assumindo os valores de 15,00 m, 5,00 m e $25,77 \mathrm{~m}$, respectivamente. 


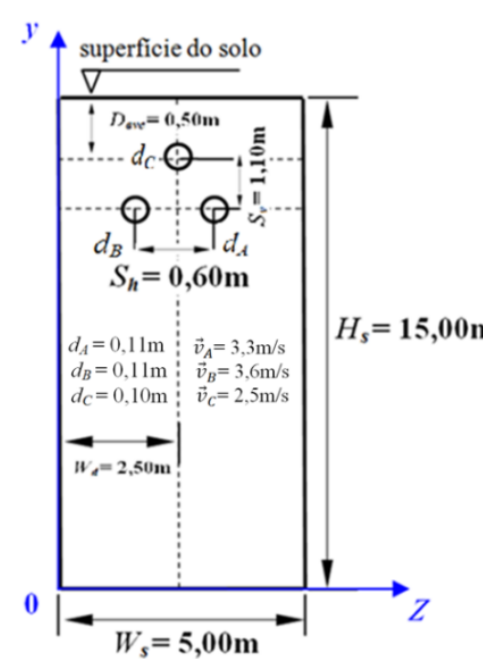

(a)

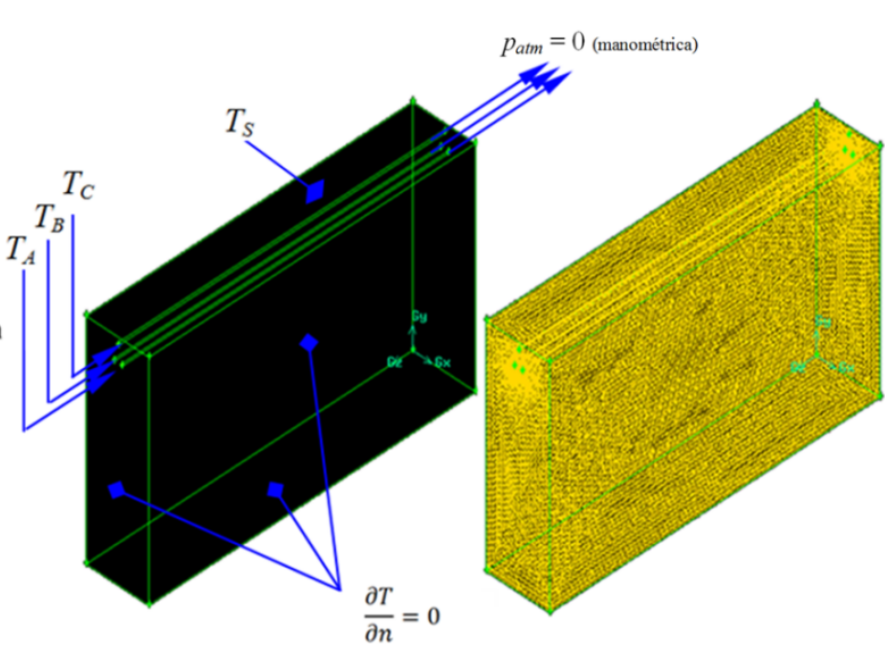

(b) (c)

Figura 1: Vista transversal (frontal) da configuração geométrica do TCSA e do domínio computacional (a), Vista em perspectiva do domínio computacional (tri-dimensional) (b) e Vista da discretização do domínio computacional em células ou volumes finitos (malha numérica) (c).

As condições de contorno, do ponto de vista térmico, para a temperatura na superfície do solo $\left(T_{S}\right)$ e para a entrada do ar nos dutos, no decorrer do tempo, foram representadas pelas variáveis $T_{A}, T_{B}$ e $T_{C}$, que, diferentemente do que vem sendo adotado em trabalhos anteriores, não foram expressas por funções definidas pelo usuário (UDF - User Defined Functions), mas sim por dados experimentais de temperaturas disponibilizados pela pesquisa feita em [13]. As temperaturas na superfície do solo no decorrer do tempo indicadas por $T_{S}$ também foram definidas a partir dos mesmos dados experimentais. Do observado na literatura, as temperaturas são modeladas por funções periódicas (do tipo senoidal), para serem inseridas no FLUENT através de UDF. Para a condição de contorno fluidodinâmica nas paredes do interior do duto foi considerada a condição de não deslizamento e de impermeabilidade. Em face da objetividade deste trabalho, foram suprimidas demais informações relacionadas às hipóteses simplificativas e condições de contorno, tanto térmica como fluidodinâmicas, no entanto, as mesmas podem ser encontradas em $[1,2]$.

A discretização do domínio computacional se deu por elementos tetraédricos gerados no GAMBIT e suas principais características podem ser identificadas na Tabela 1.

Tabela 1: Definição da malha utilizada no GAMBIT.

\begin{tabular}{c|c|c|c}
\hline Componentes & diâmetro $(\mathrm{m})$ & Tamanho do intervalo $(\mathrm{m})$ & tamanho resultante $(\mathrm{m})$ \\
\hline duto A & $d_{\mathrm{A}}=0,110$ & $d_{\mathrm{A}} / 3$ & 0,0367 \\
\hline duto B & $d_{\mathrm{B}}=0,110$ & $d_{\mathrm{B}} / 3$ & 0,0367 \\
\hline duto C & $d_{\mathrm{B}}=0,100$ & $d_{\mathrm{c}} / 3$ & 0,0333 \\
\hline Solo & ------- & $3 \times d_{\mathrm{A}}$ & 0,3300 \\
\hline
\end{tabular}

As propriedades termofísicas adotadas para o solo e o ar são dadas na Tabela 2.

Tabela 2: Propriedades termofísicas do solo e do ar.

\begin{tabular}{c|c|c|c|c}
\hline Materiais & $\begin{array}{c}\text { Massa específica } \\
\rho\left(\mathrm{kg} / \mathrm{m}^{3}\right)\end{array}$ & $\begin{array}{c}\text { Condutividade térmica } \\
k(\mathrm{~W} / \mathrm{m} \cdot \mathrm{K})\end{array}$ & $\begin{array}{c}\text { Calor específico a pressão } \\
\text { constante } c_{p}(\mathrm{~J} / \mathrm{kg} \cdot \mathrm{K})\end{array}$ & $\begin{array}{c}\text { Viscosidade } \\
\text { dinâmica } \mu \mathrm{kg} /(\mathrm{m} \cdot \mathrm{s})\end{array}$ \\
\hline Solo & 1800 & 2,1 & 1780 & ----------- \\
\hline $\mathrm{Ar}$ & 1,160 & 0,0242 & 1010 & $1,798 \times 10^{-5}$ \\
\hline
\end{tabular}

O número de Reynolds para o escoamento turbulento nos dutos A, B e C, são, respectivamente, 23419,36; 25548,38 e 16129,03 e o número de Prandtl para o ar é igual a 0,75 . 


\subsection{Modelagem matemática e numérica}

De uma forma geral, a modelagem matemática para o estudo de TCSA consiste nos princípios de conservação da massa, da quantidade de movimento e da energia. Como os escoamentos em TCSA são turbulentos, é necessário também realizar a modelagem da turbulência [1]. Para a avaliação dos campos de temperatura, o escoamento de ar nos dutos foi considerado incompressível, turbulento e descrito pelas equações de conservação de massa, momento e energia [27, 29, 30], onde se adotou, para a modelagem da turbulência, o modelo de tensões de Reynolds (RSM) [1, 29]. Quanto ao campo de temperatura no solo, este foi calculado pela equação de conservação de energia [27, 30]. Por serem de uma forma geral bem conhecidas, as equações são omitidas neste texto.

Em relação à abordagem numérica, o pré-processamento foi realizado através do software GAMBIT, onde foi construída a geometria do problema, a definição do tipo de condição de contorno e a geração da malha computacional. Com o software FLUENT, que é baseado no método dos volumes finitos (MVF), foi feito o processamento e o pós-processamento. No pósprocessamento também foi utilizado o software EXCEL. O MVF foi utilizado neste trabalho por atender, em nível de volumes elementares, os princípios de conservação da massa, do movimento e da energia [27, 28, 31]. Esse método é uma ferramenta importante no campo da CFD.

No FLUENT, foram utilizados ainda: o esquema de advecção upwind para tratar os termos advectivos e o algoritmo Coupled, de solução acoplada, para tratar do esquema pressãovelocidade. Para realização das simulações numéricas foi adotado um passo de tempo de $3.600 \mathrm{~s}$ (uma hora) em um total de 17.520 passos de tempo. A temperatura de inicialização do modelo computacional foi de $18,7^{\circ} \mathrm{C}$. Foi simulado computacionalmente dois anos de funcionamento do TCSA, no entanto, apenas os dados obtidos no segundo ano de simulação foram utilizados para análise e discussão de resultados, como também feito em [1,2].

\subsection{Ferramenta Boundary Profiles}

A ferramenta Boundary Profiles, disponível no FLUENT, pode ser utilizada para inserção de dados de perfis de fronteira (contorno) ou condições de contorno em modelos computacionais. Dessa forma, ela permite trabalhar com dados reais (dados experimentais), dados oriundos de simulação computacional iterativa (dados numéricos) ou dados obtidos por outro software computacional. Essa técnica propicia maiores oportunidades de verificação e validação de modelos computacionais, bem como ajustes de curvas e calibração de modelos computacionais de uma forma geral.

Neste trabalho, foi realizada a inclusão de dados de temperatura, a partir de um banco de dados experimentais, referenciados em [13, 14], como condições de contorno do modelo computacional para as temperaturas de entrada do ar nos dutos e na superfície do solo no decorrer do tempo. Essa ferramenta faz a leitura de arquivos de textos de formato (.txt) dispostos na forma de pares ordenados ou vetores do tipo linha (tempo $\mathrm{x}$ temperatura) em forma de tabela. Dessa forma, foram inseridos os dados experimentais de temperaturas nas entradas dos dutos indicados por $T_{A}$ (Figura 2a), $T_{B}$ (Figura 2b) e $T_{C}$ (Figura 3a), bem como dados experimentais de temperatura na superfície do solo ao longo do tempo (Figura $3 b$ ).

É importante ressaltar que como o banco de dados experimentais utilizado apresentava algumas lacunas, ou seja, sem dados registrados em certos períodos, foram inseridos valores de temperatura que seguissem a mesma tendência central da curva de temperaturas, a fim de se tornar possível a realização do presente trabalho. 


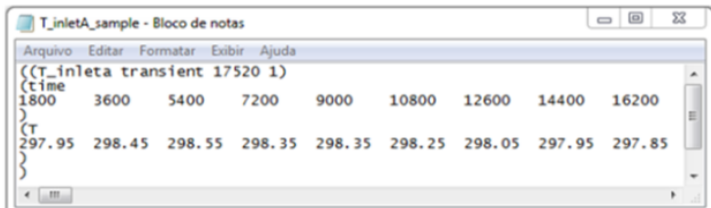

(a)

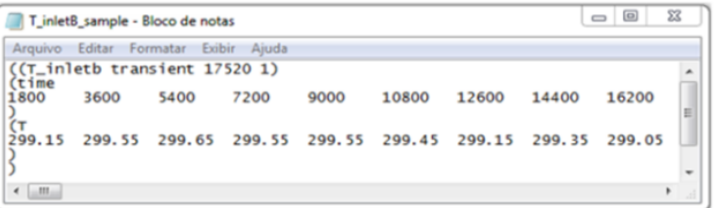

(b)

Figura 2: Ilustração dos arquivos (.txt) com dados de temperatura em função do tempo para aplicação da ferramenta Boundary Profiles referentes ao duto $A(a)$ e ao duto B (b).

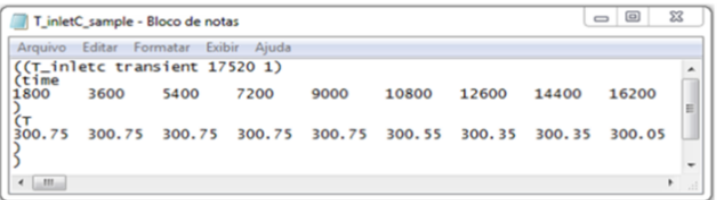

(a)

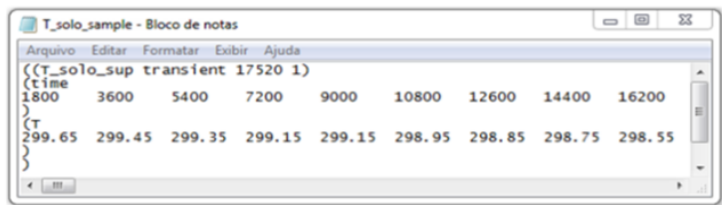

(b)

Figura 3: Ilustração dos arquivos (.txt) com dados de temperatura em função do tempo para aplicação da ferramenta Boundary Profiles referentes ao duto $C($ a) e a superfície do solo (b)

\section{RESULTADOS E DISCUSSÃO}

Os resultados apresentados consistem na verificação e validação do modelo computacional desenvolvido com base no modelo desenvolvido em [15], aprimorado em [13, 14]. Conforme a literatura, a comparação dos resultados numéricos com os resultados analíticos, se existirem, ou com outros resultados numéricos, caracteriza o processo de verificação. Por outro lado, a comparação dos resultados numéricos com os resultados experimentais é denominado de validação [27, 28].

Dessa forma, a verificação e a validação do modelo foram executadas com base em $[2,13$, 14] utilizando a ferramenta Boundary Profiles para inserção de dados de entrada no modelo computacional desenvolvido. Assim, foram realizadas comparações entre os resultados numéricos obtidos no presente trabalho com os dados numéricos e experimentais obtidos em $[13,14]$ e dados numéricos alcançados em [2].

Para analisar se os dados de entrada inseridos através da ferramenta Boundary Profile correspondem aos dados de entrada experimentais, foram inseridos sensores numéricos de temperatura na entrada dos dutos, A, B e C, bem como na superfície superior do solo. Esses sensores registraram a cada intervalo de $1.800 \mathrm{~s}$ os valores de temperatura que são apresentados, respectivamente, nas Figuras $4 \mathrm{a}, 4 \mathrm{~b}, 5 \mathrm{a}$ e $5 \mathrm{~b}$. Além disso, também foram apresentados os dados medidos experimentalmente, correspondentes à entrada do ar no trecho reto do TCSA estudado em [13, 14], além da UDF que gerou os dados de temperatura apresentado em [1].

Cabe reiterar que nas referências $[2,13,14]$ as condições de contorno de temperaturas, tanto para a superfície do solo como para as secções de entrada do ar nos dutos A, B e C, foram definidas através de UDF's, no FLUENT. Essas UDF's contém funções que definem a variação de temperatura em função do tempo, ajustadas a partir dos dados experimentais presentes em $[13,14,17]$. 


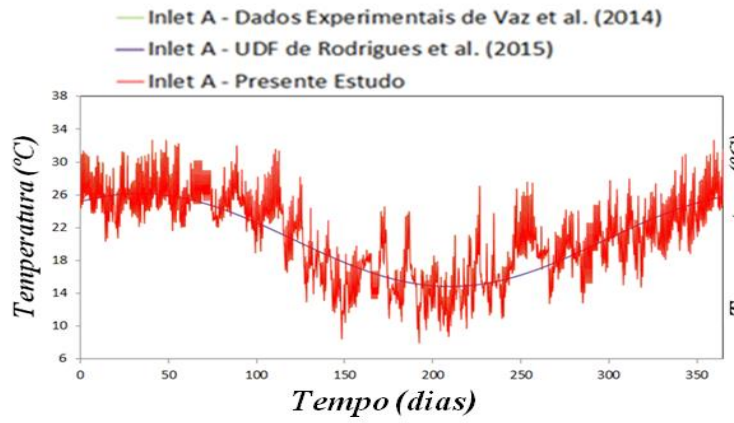

(a)

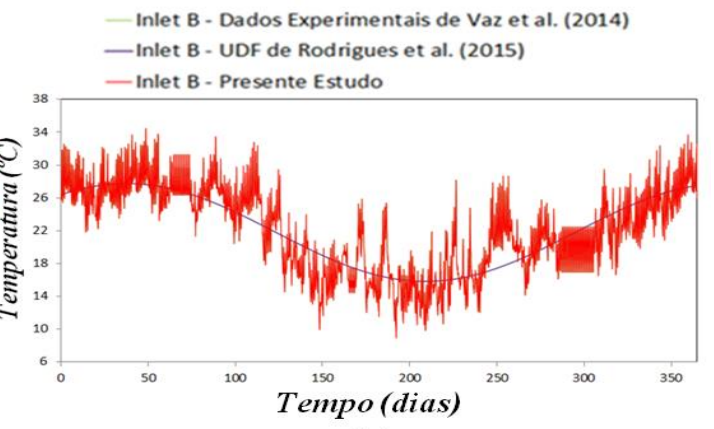

(b)

Figura 4: Comparação das variações de temperatura nas entradas do duto A (a) e do duto B (b).

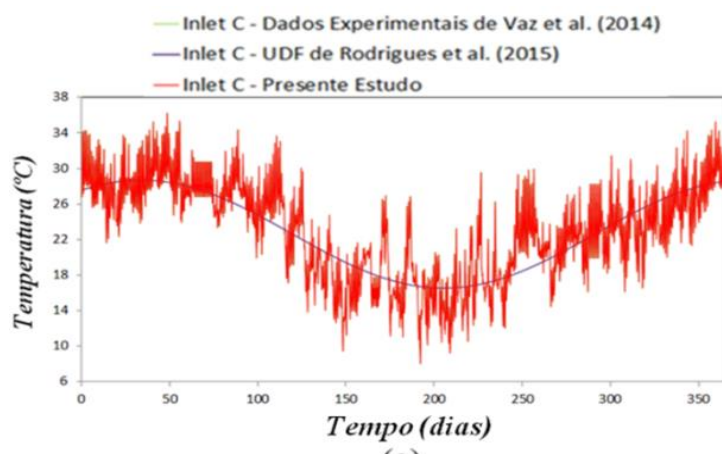

(a)

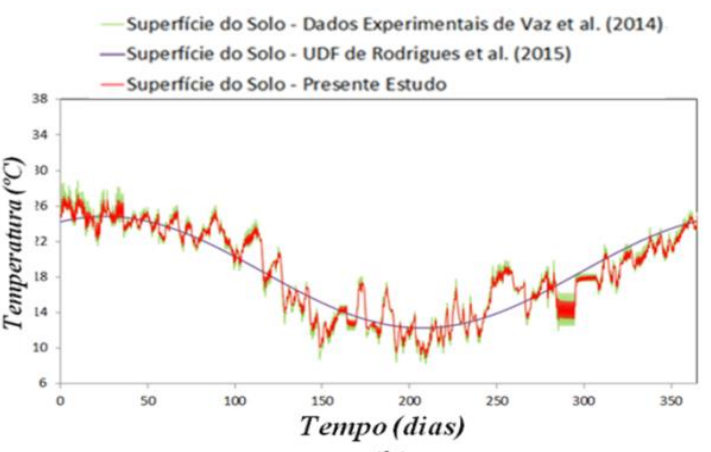

(b)

Figura 5: Comparação das variações de temperatura na entrada do duto $C(a) e$ comparação da variação de temperatura na superfície do Solo (b).

As curvas que representam a temperatura, ao longo do tempo, dos dados experimentais e simulados no presente estudo ficaram, em geral, sobrepostas, conforme apresentado nas Figuras 4a, 4b, 5a e 5b. Os dados simulados apresentaram uma correlação de 0,99 nas comparações com os dados experimentais obtidos em [13], demonstrando, consequentemente, a viabilidade na inserção de dados através de Boundary Profiles.

Nessa mesma simulação numérica, as temperaturas do ar na saída dos dutos $\mathrm{A}, \mathrm{B}$ e $\mathrm{C}$ foram também monitoradas a cada $1.800 \mathrm{~s}$, permitindo comparar os resultados obtidos no presente estudo com os dados experimentais de [13,14], bem como com os resultados numéricos obtidos nos trabalhos de $[2,13,14]$.

Nas Figuras 6a, 6b e 7, respectivamente, são comparados os resultados da temperatura do ar na saída dos dutos A, B e C com os resultados numéricos e experimentais obtidos em [13, 14], bem como os resultados numéricos obtidos em [2].

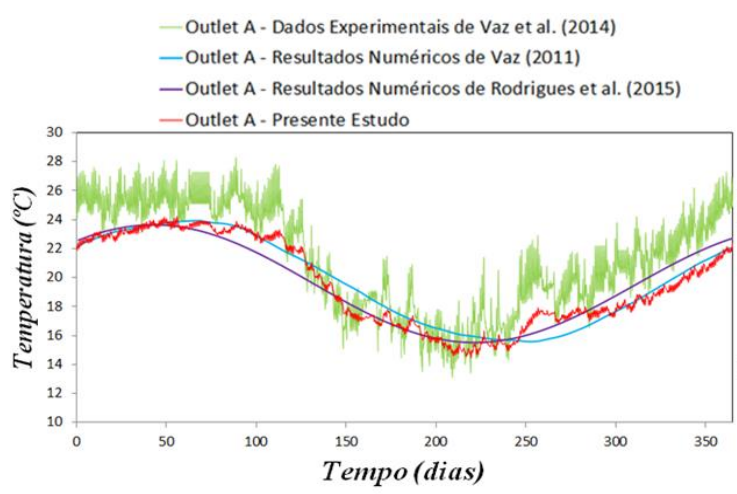

(a)

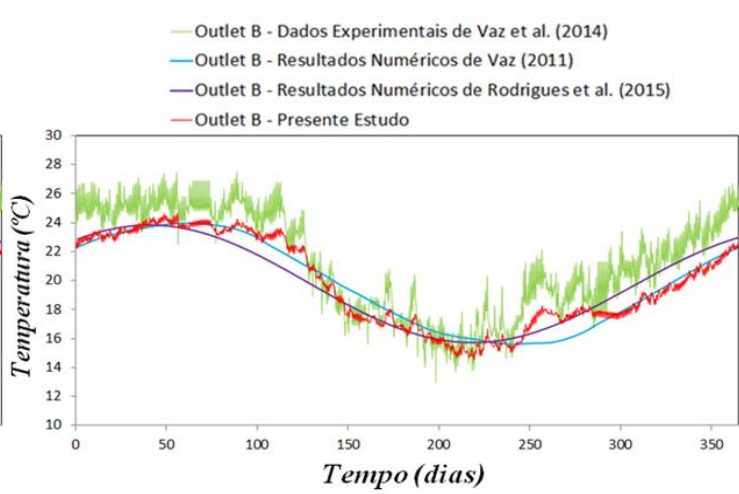

(b)

Figura 6: Comparação entre a temperatura de saída do duto A do presente estudo com resultados apresentados na literatura (a) e comparação entre a temperatura de saída do duto $B$ do presente estudo com resultados apresentados na literatura $(b)$. 


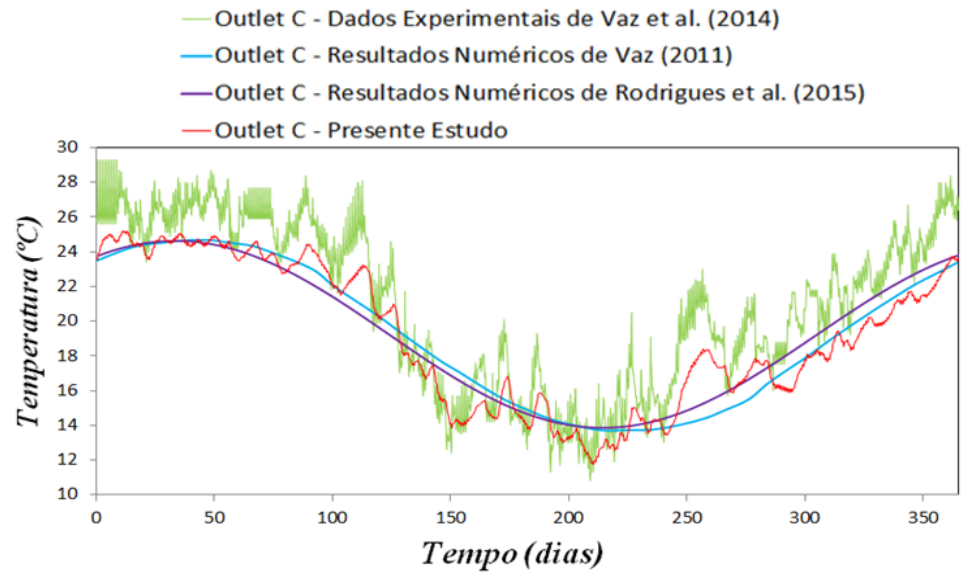

Figura 7: Comparação entre a temperatura de saída do duto $C$ do presente estudo com resultados apresentados na literatura.

As Figuras 6a, 6b e 7 também expressam que os resultados obtidos no presente estudo, em comparação com os resultados da literatura, apresentam a mesma tendência. Para comparar os resultados entre si, foram adotadas duas medidas. Primeiramente, foi calculada a raiz do valor quadrático médio (rms) [32] das diferenças (erros) entre os dados experimentais e os numéricos. Assim, o erro total foi medido por:

$$
E_{r m s}=\sqrt{\frac{1}{n} \sum_{i=1}^{n}\left(T_{\text {exp }, i}-T_{\text {num }, i}\right)^{2}},
$$

onde: $n$ é o número total de dados, enquanto $T_{\text {exp,i }}$ e $T_{n u m, i}$, em $\left({ }^{\circ} \mathrm{C}\right)$, indicam os valores das temperaturas experimentais e numéricas, respectivamente.

Embora os dados sejam discretos, as funções de temperatura que eles representam são contínuas e quadrado integráveis em qualquer intervalo de tempo. Assim, utilizando aproximações, pela regra de Simpson composta e regra do trapézio [32], o erro foi também medido pela norma, no espaço funcional $L^{2}$ [33], da diferença entre as temperaturas experimentais $T_{\text {exp }}(t)$ e numéricas $T_{\text {num }}(t)$, no intervalo de tempo $\left[t_{0}, t_{f}\right]$ considerado, isto é:

$$
E_{L^{2}}=\sqrt{\int_{t_{0}}^{t_{f}}\left(T_{\text {exp }}(t)-T_{\text {num }}(t)\right)^{2} d t} .
$$

Conforme se observa na Tabela 3, os erros numéricos no presente estudo foram, em geral, inferiores aos dos outros métodos utilizados na literatura, tanto no seu valor rms como na norma do $L^{2}$, para todos os dutos. Uma sensível melhoria ocorreu no caso do Duto C, onde o erro relativo na norma do $L^{2}$ é reduzido em aproximadamente $11 \%$ e $19 \%$, comparando-se, respectivamente com os resultados de $[2,13]$. Portanto, com base nos resultados apresentados é possível afirmar que o modelo computacional aplicando as condições de contorno de temperatura através de Boundary Profiles corresponde de maneira coerente com os resultados obtidos em estudos anteriores, aproximando-se mais dos resultados experimentais de [13, 14] do que os numéricos de $[2,13,14,15,16]$.

Tabela 3: Comparativos dos erros $E_{r m s}$ e $E_{L^{2}}$ para cada duto, considerando os dados numéricos: do presente estudo (PE); de [2]; e de [13] e [14].

\begin{tabular}{|c|c|c|c|c|c|c|}
\hline \multirow{2}{*}{ Componentes } & \multicolumn{2}{|c|}{ dados do PE } & \multicolumn{2}{c|}{ dados de [2] } & \multicolumn{2}{c|}{ dados de [13] e [14] } \\
\cline { 2 - 7 } & $E_{r m s}\left({ }^{\circ} \mathrm{C}\right)$ & $E_{L^{2}}\left({ }^{\circ} \mathrm{C} \cdot \mathrm{dia}^{1 / 2}\right)$ & $E_{r m s}\left({ }^{\circ} \mathrm{C}\right)$ & $E_{L^{2}}\left({ }^{\circ} \mathrm{C} \cdot \mathrm{dia}^{1 / 2}\right)$ & $E_{r m s}\left({ }^{\circ} \mathrm{C}\right)$ & $E_{L^{2}}\left({ }^{\circ} \mathrm{C} \cdot \mathrm{dia}^{1 / 2}\right)$ \\
\hline duto A & 2,2 & 42,0 & 2,2 & 42,9 & 2,4 & 45,3 \\
\hline duto B & 1,8 & 34,8 & 1,9 & 37,0 & 2,1 & 40,9 \\
\hline duto C & 2,2 & 42,5 & 2,5 & 47,3 & 2,7 & 50,6 \\
\hline
\end{tabular}




\section{CONCLUSÕES}

A contribuição mais significativa deste trabalho foi a introdução de dados obtidos experimentalmente como condições de contorno para o modelo de TCSA desenvolvido através da utilização da ferramenta Boundary Profiles, ao invés de UDF's que vinham sendo utilizadas em diversos trabalhos referenciados anteriormente. Esse método permite a variação da temperatura, ou outra variável qualquer, para dado instante de tempo, apresentando resultados mais precisos ao inserir dados experimentais no modelo computacional. Isso foi comprovado a partir dos valores de temperaturas obtidos experimentalmente e que foram impostos como condição de contorno no modelo computacional. Ao monitorar, ao longo do tempo, percebeu-se que os dados experimentais foram reproduzidos mais precisamente no modelo computacional, observando-se reduções nos erros numéricos de até $19 \%$.

Através da análise, comparação e discussão apresentadas neste trabalho, conclui-se que o os resultados numéricos correspondem de forma semelhante aos dados obtidos experimentalmente, porém é perceptível que a variação das amplitudes das temperaturas na saída do TCSA apresentam determinado amortecimento, quando comparadas com os resultados experimentais já referenciados. Entretanto, destaca-se que os resultados obtidos no presente estudo apresentam uma boa concordância com os resultados obtidos numericamente em [2, 13, 14]. Portanto, acredita-se que essa diminuição na amplitude das temperaturas de saída do TCSA, bem como a defasagem na magnitude das temperaturas, observadas em relação aos dados experimentais, pode estar relacionada com algum parâmetro adotado na modelagem computacional utilizada.

Com o intuito de aproximar os resultados numéricos dos experimentais, estão sendo realizadas tentativas utilizando o modelo de turbulência $k-\varepsilon$ com resíduos padrões e com resíduos reduzidos. Resultados preliminares apontam a redução do tempo de processamento utilizando o modelo de turbulência $k-\varepsilon$ ao invés do modelo RSM utilizado neste trabalho, tendo impactos não significativos nos resultados finais. O modelo de turbulência $k$ - $\omega$ também será investigado em estudos futuros.

\section{AGRADECIMENTOS}

L. A. Isoldi agradece ao CNPq pelo Projeto Universal (processo: 445558/2014-8). E. D. dos Santos, L. A. Isoldi e L. A. O. Rocha agradecem ao CNPq por suas bolsas de Produtividade em Pesquisa. R. S. Brum agradece ao CNPq por sua bolsa de doutorado.

\section{REFERÊNCIAS BIBLIOGRÁFICAS}

1. Rodrigues MK, Dos Santos ED, Isoldi LA. Numerical Analysis and Constructal Design of Earth-Air Heat Exchanger - Mathematical Methods for Engineering. Saarbrücken: Lap Lambert Academic Publishing; 2015. 167 p.

2. Rodrigues MK, Brum RS, Vaz J, Rocha LAO, Dos Santos ED, Isoldi LA. Numerical investigation about the improvement of the thermal potential of an Earth-Air Heat Exchanger (EAHE) employing the Constructal Design method. Renew Energ. 2015 Aug;80(1):538-51,doi: 10.1016/j.renene.2015.02.041.

3. Bharadwaj SS, Bansal NK. Temperature distribution inside ground for various surface conditions. Build Environ. 1981 Jan;16(3):183-92,doi: 10.1016/0360-1323(81)90012-3.

4. Givoni B, Katz L. Earth temperatures and underground buildings. Energ Buildings. 1985 Feb; 8(1):15-25, doi: 10.1016/0378-7788(85)90011-8.

5. Mihalakakou G, Santamouris M, Asimakopoulos D, Argiriou A. On the ground temperature below buildings. Sol Energy. 1995 Nov;55(9):355-62,doi: 10.1016/0038-092X(95)00060-5.

6. Jacovides CP, Mihalakakou G, Santamouris M, Lewis JO. On the ground temperature profile for passive cooling applications in buildings. Sol Energy. 1996 Sep;57(3):167-75,doi: 10.1016/S0038092X(96)00072-2.

7. Mihalakakou G, Santamouris M, Lewis JO, Asimakopoulos D. On the application of the energy balance equation to predict ground temperature profiles. Sol Energy. 1997 Mar;60(3):181-90,doi: 10.1016/S0038-092X(97)00012-1. 
8. Mihalakakou G, Santamouris M, Asimakopoulos D. Modeling the thermal performance of earth-to-air heat exchangers. Sol Energy. 1994 Sep;53(3):301-5,doi: 10.1016/0038-092X(94)90636-X.

9. Santamouris M, Mihalakakou G, Argiriou A, Asimakopoulos D. On the performance of buildings coupled with earth to air heat exchangers. Sol Energy. 1995 Jun;54(6):375-80,doi: 10.1016/0038092X(95)00016-K.

10. De Paepe M, Janssens A. Thermo-hydraulic design of earth-air heat exchangers. Energ Buildings. 2003 May;35(4):389-97,doi: 10.1016/S0378-7788(02)00113-5.

11. Trzaski A, Zawada B. The influence of environmental and geometrical factors on air- ground tube heat exchanger energy efficiency. Build Environ. 2011 Jul;46(7):1436-44,doi: 10.1016/j.buildenv.2011.01.010.

12. Ascione F, Bellia L, Minichiello F. Earth-to-air heat exchangers for Italian climates. Renew Energ. 2011 Aug;36(8):2177-88,doi: 10.1016/j.renene.2011.01.013.

13. Vaz J. Estudo experimental e numérico sobre o uso do solo como reservatório de energia para o aquecimento e resfriamento de ambientes edificados [Tese]. Porto Alegre (RS): Universidade Federal do Rio Grande do Sul; 2011. 237 p.

14. Vaz J, Sattler MA, Dos Santos ED, Isoldi LA. Experimental and Numerical Analysis of an Earth-Air Heat Exchanger. Energ Buildings. 2011 Sep;43(9):2476-82,doi: 10.1016/j.enbuild.2011.06.003.

15. Brum RS, Rocha LAO, Vaz J, Dos Santos ED, Isoldi LA. Development of Simplified Numerical Model for Evaluation of the Influence of Soil-Air Heat Exchanger Installation Depth over its Thermal Potential. Int J Adv Renew Energ Res. 2012 Jan;1(1):505-14.

16. Brum RS, Vaz J, Rocha LAO, Dos Santos ED, Isoldi LA. A New Computational Modeling to Predict the Behavior of Earth-Air Heat Exchangers. Energ Buildings. 2013 Sep;64(1):395-402,doi: 10.1016/j.enbuild.2013.05.032.

17. Vaz J, Sattler MA, Brum RS, Dos Santos ED, Isoldi LA. An experimental study on the use of earth-air heat exchangers (EAHE). Energ Buildings. 2014 Apr;72(1):122-31,doi: 10.1016/j.enbuild.2013.12.009.

18. Rodrigues MK, Souza JA, Vaz J, Junior ICA, Rocha LAO, Brum RS, Dos Santos ED, Isoldi LA. Estudo numérico da influência de condições de contorno na modelagem computacional de trocador de calor solo-ar. Sci Plena. 2015 Mar;11(8):1-10,doi: 10.14808/sci.plena.2015.081334.

19. Do SL, Baltazar JC, Haberl J. Potential cooling savings from a ground-coupled return-air duct system for residential buildings in hot and humid climates. Energ Buildings. 2015 Sep;103(1):206-15,doi: 10.1016/j.enbuild.2015.05.043.

20. Ferraz JG. Modelagem computacional de trocador de calor solo-ar comparando diferentes modelagens para a turbulência [Dissertação]. Rio Grande (RS): Universidade Federal do Rio Grande; 2015. 96 p.

21. Kaushal M, Dhiman P, Singh S, Patel H. Finite volume and response surface methodology based performance prediction and optimization of a hybrid earth to air tunnel heat exchanger. Energ Buildings. 2015 Oct;104(1):25-35,doi: 10.1016/j.enbuild.2015.07.014.

22. Gan G. Simulation of dynamic interactions of the earth-air heat exchanger with soil and atmosphere for preheating of ventilation air. Appl Energ. 2015 Nov;158(1):118-32,doi: 10.1016/j.apenergy.2015.08.081.

23. Nunes B. Modelagem computacional aplicada ao estudo de trocadores de calor solo-ar com configuração geométrica complexa [Dissertação]. Rio Grande (RS): Universidade Federal do Rio Grande; 2015. $152 \mathrm{p}$.

24. Nunes BR, Ferraz JG, Kipper R., Rodrigues MK, Brum RS, Souza JA, Rocha, LAO, Dos Santos ED, Isoldi LA. Modelagem computacional aplicada ao estudo de um trocador de calor solo-ar com diferentes configurações geométricas. Sci Plena. 2015 Jan;11(8):1-10,doi: 10.14808/sci.plena.2015.081305.

25. Ferraz JG, Nunes BR, Rodrigues MK, Brum RS, Souza JA, Rocha LAO, Isoldi, LA. Dos Santos ED. Modelagem computacional de um trocador de calor solo-ar empregando diferentes modelagens para turbulência. Sci Plena. 2015 Jan;11(8):1-10,doi: 10.14808/sci.plena.2015.081314.

26. Marzarotto C, Nunes B, Rodrigues MK, Souza JA, Rocha, LAO, Brum RS, Dos Santos ED, Isoldi, LA. Análise numérica da influência de parâmetros operacionais e construtivos no funcionamento de trocadores de calor solo-ar. Sci Plena. 2015 Jan;11(8):1-11,doi: 10.14808/sci.plena.2015.081304.

27. Versteeg H, Malalasekera W. An Introduction to Computational Fluid Dynamics: The Finite Volume Method. London: Pearson Education Limited; 2007. 520 p.

28. Maliska CR. Transferência de Calor e Mecânica dos Fluidos Computacional. Rio de Janeiro: LTC; 2012. 472p.

29. Wilcox DC. Turbulence Modeling For CFD. California: DCW Industries; 2006. 522 p.

30. Incropera FP, Dewitt DP, Bergman, TL, Lavine AS. Fundamentos de transferência de calor e de massa. Rio de Janeiro: LTC; 2008. 644 p. 
31. Patankar SV. Numerical Heat Transfer and Fluid Flow. New York: McGraw-Hill; 1980. 214 p.

32. Burden RL, Faires JD. Análise numérica - Tradução da 8a. edição norte-americana. São Paulo: Cengage Learning; 2008. 736 p.

33. Reddy BD. Introductory functional analysis: with applications to boundary value problems and finite elements. New York: Springer-Verlag; 1998. 472 p. 